\title{
CORRIGENDUM
}

\section{Brg-1 targeting of novel miR550a-5p/RNF43/Wnt signaling axis regulates colorectal cancer metastasis}

G Wang, Y Fu, X Yang, X Luo, J Wang, J Gong and J Hu

Oncogene (2017) 36, 5915; doi:10.1038/onc.2017.317; published online 28 August 2017

Correction to: Oncogene (2016) 35, 651-661; doi:10.1038/onc. 2015.124; published online 11 May 2015

The authors noted after publication that the listing of author institution "Cancer Research Institute, Tongji Medical College, Huazhong University of Science and Technology,
Wuhan, China' was incorrect. This should be replaced by "Cancer Research Institute, Tongji Hospital, Tongji Medical College, Huazhong University of Science and Technology, Wuhan, China'.

The authors apologise for any inconvenience caused by this error. 